\title{
Coordinates Tracking Intrusion Surveillance Technology using Infrared Thermal Imagings
}

\author{
by Choi. M. Y.*, Kim. S. U.*, Kwon. K. A.*, Park. J. H.*, Ahn. B. Y.*
}

\author{
* Safety Measurement Center, Korea Research Institute of Standards and Science, Daejeon, Korea, \\ mychoi@kriss.re.kr
}

\begin{abstract}
Currently, our nuclear plants are equipped with various physical protection facilities. However, integration of IT and high-tech sensing technology is inadequate and real-time monitoring system and most of video security and detection systems are limited with only statically recording function and these are all done manually. Inadequate management know-how is further worsening the situation. Therefore, our research is trying to suggest a real-time monitoring system that can make use of infrared thermal imaging integrating detection system with physical protection system that performs area observation and analysis, automatic recognition of security status and report to the person in charge replacing manly work. It contributes to the innovative improvement in physical protection system technology as well as the international competitiveness of enterprises.
\end{abstract}

\section{Introduction}

Currently, Korea's nuclear plants are equipped with various physical protection facilities. However, integration of IT and high-tech sensing technology is inadequate. In addition, because most of sensors used for physical protection are being imported, purchase and maintenance are resulting in severe loss. Therefore, our research is trying to suggest a real-time monitoring system that can make use of infrared thermal imaging integrating detection system with physical protection system that performs area observation and analysis, automatic recognition of security status and report to the person in charge replacing manly work.

\section{Study Contents}

\subsection{Measurement of Infrared Thermal Imaging}

An infrared thermal imaging integration detection system is a device that indicates a temperature and color distribution's thermal imaging by detecting invisible far-infrared radiation energy through a contact method. Also, it is a device that measures various far-infrared radiation applied products, raw materials' heat distribution, energy conservation, overload, availability of insulation, and effects of thermal bridges and applied products on human body.

The measurement of infrared thermal imaging enables relative comparison between far-infrared energy distribution, temperatures for certain parts and the overall product's average temperature and has the merit of observing them in real time.

Infrared thermal imaging is not affected by the product's temperature, since it measures the surface temperature for a wide area through a contactless method. Therefore, it infrared thermal imaging allows for safety, speed, and easy measurement at uneasy access. Also, infrared thermal imaging has no limitation in terms of distance and embodies accurate imaging even under darkness.

\subsection{Coordinate-Tracking Technology Using Infrared Thermal Imaging}

Today's security monitoring system does not allow for real-time implementation due to the use of an excessively complex algorithm and thus it cannot recognize and track a person without any movement. It is possible to track and recognize a person through a binary code without any complex algorithm by using the dynamic zoom control tracking method of CCD Camera making use of Thermal Imaging Coordinates suggested in this study. Also, the camera has the merits of Identity Verification and Resolution, Day and Night Tracking Accuracy, Identify Verification Monitoring Distance, System Establishment Cost.

\subsection{Computer Simulation Design}



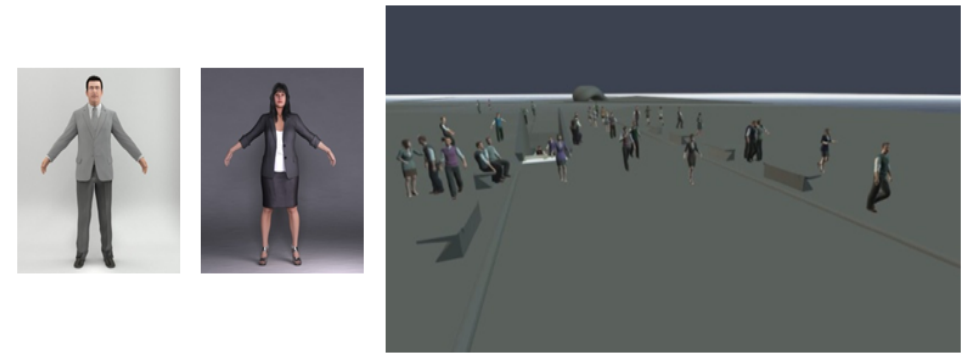

Fig. 1 Computer simulation

By utilizing the human modeling of average size Korean men and women, the PTZ system is organized within the computer space and the Thermal Imaging coordinates-tracking algorithm is embodied.

\subsection{Coordinate Transformation Processing (Calibration)}

By coordinating the IR images $(640 \times 480)$ from $45 \mathrm{~mm}$ lens with the calibration function suggested in this very research, the Pixel coordinates are transformed into World coordinates and then presented onto the PTZ coordinates to obtain images through PTZ controls of a tracing camera. It is possible for a transformation of IR Image $(640 \times 480)$ coordinates into World coordinates by using the functional formula(1) and (2) suggested in this very research for coordinates that are plotted differently for each installation height $h(2 \mathrm{~m} \sim 10 \mathrm{~m})$ of the camera.

$$
\begin{aligned}
& -\mathrm{h} / \tan (-0.125 \mathrm{X}+30.125){ }^{\star} \operatorname{Cos}\left(\mathrm{Y}^{\star} 0.002182\right) \\
& -\mathrm{h} / \tan (-0.125 \mathrm{X}+30.125) \star \operatorname{SIN}\left(\mathrm{Y}^{\star} 0.002182\right)
\end{aligned}
$$

\subsection{PTZ Optimum Speed Design}

A camera that has received the real world coordinates obtained by the thermal imaging camera uses Pan, Tilt, and Zoom functions in order to acquire Identity Verification Domain. In this very research, the maximum speed for a human is set at $11 \mathrm{~m} / \mathrm{s}$ for the design of PTZ optimum speed
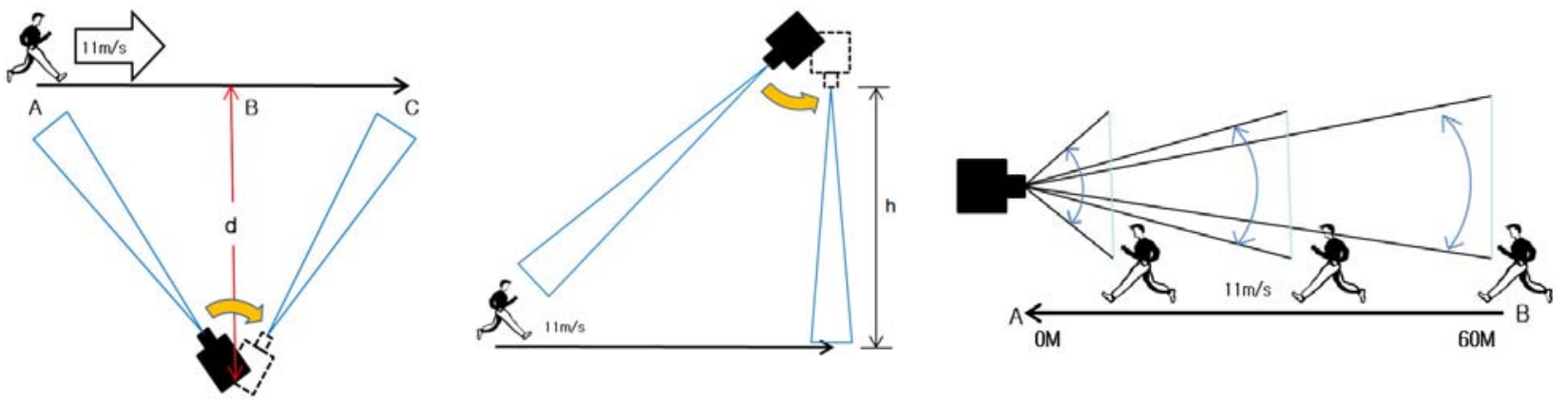

Fig. 2 Computer simulation

Table 1. Maximum panning, tilting speed according to the distance between the target

\begin{tabular}{|c|c|c|c|c|c|c|c|c|c|}
\hline $\mathrm{d}(\mathrm{m})$ & 2 & 3 & 4 & 5 & 6 & 7 & 8 & 9 & 10 \\
\hline $\begin{array}{c}\text { Maximum Panning and Tilting } \\
\text { Velocity (deg/s) }\end{array}$ & 314.8 & 210 & 157.5 & 126 & 105 & 90 & 78.8 & 70 & 63 \\
\hline
\end{tabular}

Table 2. Maximum zoom speed according to the distance between the target

\begin{tabular}{|c|c|c|c|c|c|c|c|c|c|c|c|}
\hline $\mathrm{d}(\mathrm{m})$ & $5-10$ & $10-15$ & $15-20$ & $20-25$ & $25-30$ & $30-35$ & $35-40$ & $40-45$ & $45-50$ & $50-55$ & $55-60$ \\
\hline $\begin{array}{c}\text { Zoom } \\
\text { Velocity } \\
\text { (deg/s) }\end{array}$ & $\mathbf{5 7 . 0 2}$ & 20.99 & $10-74$ & 6.51 & 4.36 & 3.12 & 2.33 & 1.83 & 1.47 & 1.19 & 1.01 \\
\hline
\end{tabular}

2.6 Maximum View Angle and Distance for face recognition 
The minimum required resolution was 576 pixels in vertical for face identification and 290 pixels in vertical for face recognition

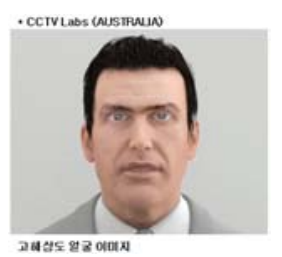

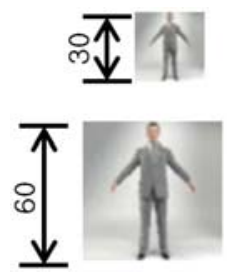

Fig. 3 Pixel for face recognition

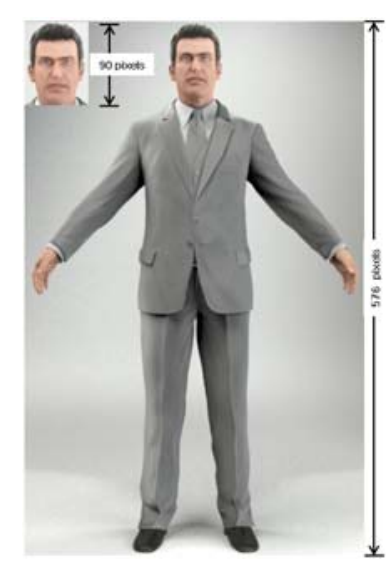

\subsection{Shutter Speed for No Blur}

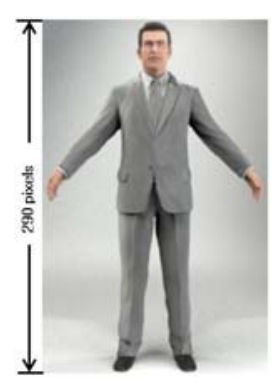

Applied settings for IR Camera $\left(640 \times 480\right.$, standard view angle $25 \mathrm{~mm}\left(\mathrm{H} \times \mathrm{V}\right.$, degree : $\left.\left.71.508^{\circ} \times 56.738^{\circ}\right)\right)$. After calculating the 1 Pixel real distance in accordance with Theory of Proportion, the calculation was carried out by setting the human maximum speed at $11 \mathrm{~m} / \mathrm{s}$. Each shutter speed is calculated for different distances between the focal point and the object of $27 \mathrm{~m}, 40 \mathrm{~m}, 80 \mathrm{~m}$, and $100 \mathrm{~m}$, respectively.

\section{Conclusion}

1) By applying the human recognition and tracing utilized by the thermal imaging suggested in this very research, a convenient and rapid human recognition based on binary-code will be possible without neither a complicated algorithm nor a mathematical approach.

2) The PTZ maximum Pan speed for human tracing turned out to be $314.8 \mathrm{deg} / \mathrm{s}$, the maximum Tilt speed at 314.8deg/s, and the maximum Zoom speed at $57.02 \mathrm{deg} / \mathrm{s}$ from the PTZ Optimum Speed Design

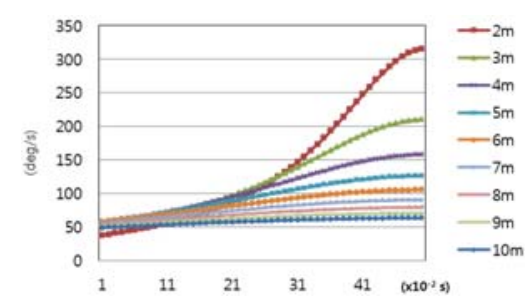

Maximum Panning speed

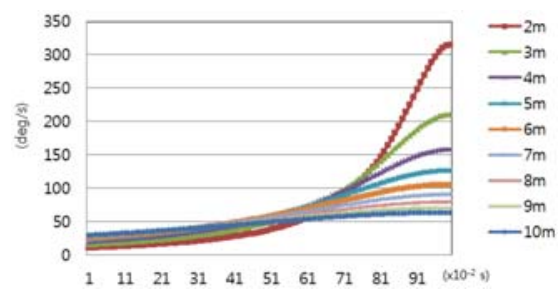

Maximum Tilting speed

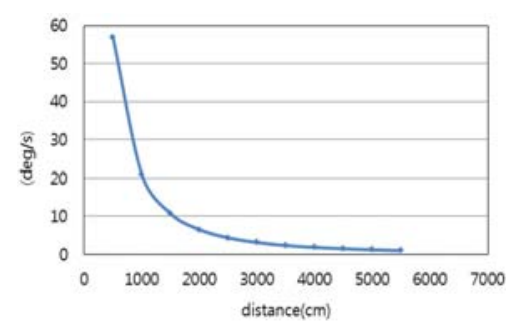

Maximum Zooming speed

Fig. 4 Maximum PTZ speed

3) As a result for the calculation of the shutter speed for No Blur, 1/181sec, 1/122sec, 1/61sec, and 1/49sec were shown for distances between the focal point and the object of $27 \mathrm{~m}, 40 \mathrm{~m}, 80 \mathrm{~m}$, and $100 \mathrm{~m}$, respectively

Table 3 Shutter speed for No Blur according to the distance between the target

\begin{tabular}{|c|c|c|c|c|}
\hline \multicolumn{5}{|c|}{$640 \times 480$ ( Pixel $P_{x}=25 \mu \mathrm{m}$ ) } \\
\hline$d(m)$ & 27 & 40 & 80 & 100 \\
\hline Sutter Speed & $1 / 181 \mathrm{sec}$ & $1 / 122 \mathrm{sec}$ & $1 / 61 \mathrm{sec}$ & $1 / 49 \mathrm{sec}$ \\
\hline
\end{tabular}




\section{Acknowledgement}

This work was supported by the NAP(National Agenda Project) of the Korea Research Council of Fundamental Science \& Technology(IP2010-0028) and a project of consignment development of the Korea Institute of Nuclear nonproliferation and Control(IP2011-0004).

\section{REFERENCES}

[1] Chang-geun Shin., "Development of Moving Object Tracking Algorithm using hue Distribution of Multi-video Camera Image" Cheongju University PHD 2004.

[2] Gang-mo Yim., "Study on Real-time Tracking of Moving Object by the Decimation-in-time Search Algorithm" Cheongju University PHD 2005.

[3] Bu-il Park., "Tracking Soccer Players using Multi-Camera" Sogang University, Graduate School 2004. 